\title{
A QUASI-NEWTON APPROACH TO IDENTIFICATION OF A PARABOLIC SYSTEM
}

\author{
WENHUAN YU'
}

(Received 3 January 1996; revised 20 January 1997)

\begin{abstract}
A quasi-Newton method (QNM) in infinite-dimensional spaces for identifying parameters involved in distributed parameter systems is presented in this paper. Next, the linear convergence of a sequence generated by the QNM algorithm is also proved. We apply the QNM algorithm to an identification problem for a nonlinear parabolic partial differential equation to illustrate the efficiency of the QNM algorithm.
\end{abstract}

\section{Introduction}

Quasi-Newton methods play an important role in numerically solving optimization problems on the Euclidean spaces. But few papers discuss these methods in identification of infinite-dimensional systems.

Formulating parameter estimation problems as constrained, regularized optimization problems, Kunisch et al. [13] investigated the reduced SQP (Sequential Quadratic Programming) methods with BFGS (Broyden-Flecher-Goldfarb-Shanno) update for the identification of an elliptic system.

In this paper we formulate an identification problem as an unconstrained optimization one. We suggest a Quasi-Newton Method (QNM) to solve an unconstrained optimization problem in Section 2. Following Broyden et al. [3] and using the HilbertSchmidt class defined in [7], we prove that the approximate sequence generated by the QNM procedure converges to the optimal element of the optimization problem if the latter exists. In Section 3 we apply the QNM algorithm to estimating a coefficient appearing in a nonlinear parabolic partial differential equation and we prove that the assumptions, which ensure the convergence of the approximate sequence obtained by the QNM algorithm, are satisfied. Finally, we illustrate a numerical example to show the efficiency of the QNM algorithm.

\footnotetext{
'Department of Mathematics, Tianjin University, Tianjin 300072, People's Republic of China. (C) Alistralian Mathematical Society, 1998, Serial-fee code 0334-2700/98
} 
There are many papers dealing with the parameter identification problem for distributed parameter systems. Methods of solving some of those problems are listed in the following:

(1) the gradient or conjugate gradient methods, for example, Chavent et al. [4], Seinfeld et al. [17, 16], and Yu [18], which need to compute the derivative maps of the operators described by partial differential equations;

(2) the generalized pulse spectrum technique (GPST), for example, Chen et al. [5, 22];

(3) the finite-dimensional approximate modal methods, for example, Banks et al. [1, 2];

(4) the regularization methods, for example, Yu et al. [19, 20, 21];

(5) the sequential quadratic programming (SQP) methods, for example, Kunisch and Sachs [13] and Huang et al. [9];

(6) the Lagrangian method, for example, Ito and Kunisch [10, 11];

(7) Quasi-Newton methods for solving unconstrained optimal control problems, for example, Kelley and Sachs [13].

Finally, it should be pointed out that proving a superlinear rate of convergence for quasi-Newton methods in infinite-dimensional spaces is not trivial as it is in finite-dimensional spaces. The Q-superlinear convergence for the above-mentioned sequence can be obtained under an additional condition assumed by Griewank [8].

The QNM algorithm presented in this paper can also be applied to identification problems of other PDS's.

\section{A quasi-Newton method in Hilbert spaces}

We consider the following unconstrained optimization problem (UOP):

$$
\text { minimize } f(x) \text {, }
$$

where $f: H \rightarrow \mathbb{B}$, and $H$ is a Hilbert space. A point $x^{*}$ is called optimal for UOP if $f$ attains a local minimum at $x^{*}$.

It is well-known that the necessary condition for $x^{*}$ being optimal is

$$
f^{\prime}\left(x^{*}\right)=0,
$$

where $f^{\prime}\left(x^{*}\right) \in \mathscr{L}(H ; \mathbb{R}) \equiv H^{\prime}$ is the Fréchet derivative of $f$ at $x^{*}, \mathscr{L}(X ; Y)$ denotes the space of bounded linear operators from a Banach space $X$ to a Banach space $Y$ with the operator norm and $H^{\prime}$ is the adjoint space of $H$.

If $f: \mathbb{R}^{n} \rightarrow \mathbb{R}$, one frequently uses quasi-Newton methods for solving UOP because of their high efficacy. So, we use a quasi-Newton method for solving UOP as 
an iterative scheme which generates the sequences $\left\{x_{k}\right\}$ and $\left\{A_{k}\right\}$ from the formulas

$$
\begin{aligned}
A_{k} s_{k} & =-f^{\prime}\left(x_{k}\right), \\
x_{k+1} & =x_{k}+s_{k}, \\
y_{k} & =f^{\prime}\left(x_{k+1}\right)-f^{\prime}\left(x_{k}\right), \\
A_{k+1} & =A_{k}+y_{k}\left\langle y_{k}, \cdot\right\rangle /\left\langle y_{k}, s_{k}\right\rangle-A_{k} s_{k}\left\langle A_{k} s_{k}, \cdot\right\rangle /\left\langle A_{k} s_{k}, s_{k}\right\rangle,
\end{aligned}
$$

where $x_{0}$ and $A_{0}$ are given, $\langle\cdot, \cdot\rangle$ is the dual product between $H^{\prime}$ and $H$, and for any $y \in H^{\prime}$ the operator $\langle y, \cdot\rangle: H \rightarrow \mathbb{R}$ is defined by

$$
\langle y, \cdot\rangle x \equiv\langle y, x\rangle, \quad \forall x \in H
$$

Obviously, $A_{k} \in \mathscr{L}\left(H, H^{\prime}\right)$ and $y_{k}, f^{\prime}\left(x_{k}\right) \in H^{\prime}$.

The above algorithm is just a BFGS formula in a Hilbert space. If set $B_{k} \equiv A_{k}^{-1}$, then by the Sherman-Morrison-Woodbury formula, we obtain

$$
B_{k+1}=B_{k}+\frac{\left(s_{k}-B_{k} y_{k}\right)\left\langle\cdot, s_{k}\right\rangle+s_{k}\left\langle\cdot, s_{k}-B_{k} y_{k}\right\rangle}{\left\langle y_{k}, s_{k}\right\rangle}-\frac{\left\langle y_{k}, s_{k}-B_{k} y_{k}\right\rangle}{\left\langle y_{k}, s_{k}\right\rangle^{2}} s_{k}\left\langle\cdot, s_{k}\right\rangle,
$$

and $B_{k} \in \mathscr{L}\left(H^{\prime} ; H\right)$.

In this paper $K: H \rightarrow H^{\prime}$ is the canonical isometry, that is, for any $x \in H$ $K x \in H^{\prime}$ and

$$
\langle K x, s\rangle=(x, s), \quad \forall s \in H,
$$

$(\cdot, \cdot)$ and $((\cdot, \cdot))$ are the inner products of $H$ and $H^{\prime}$, respectively.

The following definition can be found in [7].

DEFINITION 2.1. Let $\mathscr{B}_{0}\left(H ; H^{\prime}\right)$ be the class of all compact operators on $H$ and $H^{\prime}$. For any $T \in \mathscr{B}_{0}\left(H ; H^{\prime}\right)$, define

$$
\|T\|_{2} \equiv\left(\sum_{k=1}^{\infty}\left\|T \phi_{k}\right\|^{2}\right)^{1 / 2},
$$

where $\|\cdot\|$ is the norm of $H^{\prime}$ and $\left\{\phi_{k}\right\}$ is a complete orthonormal family in $H$. If the series in the right-hand side does not converge, set $\|T\|_{2}=+\infty$. Moreover, $\|T\|_{2}$ is independent of the choice of the complete orthonormal family $\left\{\phi_{k}\right\}$ in (2.8). $\|T\|_{2}$ is called the Schmidt norm of $T$. The subset of $\mathscr{B}_{0}\left(H ; H^{\prime}\right)$ consisting of all $T$ with $\|T\|_{2}<+\infty$ is called the Hilbert-Schmidt class, which is denoted by $\mathscr{B}_{2}\left(H ; H^{\prime}\right)$.

$\mathscr{B}_{2}\left(H ; H^{\prime}\right)$ is a Banach space with the norm $\|\cdot\|_{2}$.

DEFINITION 2.2. For any $T \in \mathscr{B}_{2}\left(H^{\prime} ; H\right)$, define the norm

$$
\|T\|_{M} \equiv\|K M T K M\|_{2},
$$

where $M: H \rightarrow H$ is positive and self-adjoint.

$\mathscr{B}_{2}\left(H^{\prime} ; H\right)$ is a Banach space with the norm $\|\cdot\|_{M}$. 
Obviously, we have the following properties.

LEMMA 2.1. If $T \in \mathscr{B}_{2}\left(H ; H^{\prime}\right), S_{1} \in \mathscr{L}(H)$, and $S_{2} \in \mathscr{L}\left(H^{\prime}\right)$, where $\mathscr{L}(X)$ is the space of bounded linear operators with the operator norm from $X$ to $X$, then $S_{2} T$, $T S_{1} \in \mathscr{B}_{2}\left(H ; H^{\prime}\right)$ and

$$
\left\|T S_{1}\right\|_{2} \leq\left\|S_{1}\right\|\|T\|_{2}, \quad\left\|S_{2} T\right\|_{2} \leq\left\|S_{2}\right\|\|T\|_{2} .
$$

Moreover, there are positive constants $\eta_{1}$ and $\eta_{2}$ with $\eta_{2} \geq 1$ such that $\forall T \in$ $\mathscr{B}_{2}\left(H ; H^{\prime}\right)$

$$
\eta_{1}\|T\|_{M} \leq\|T\| \leq \eta_{2}\|T\|_{M}
$$

In this paper we always suppose that the following assumptions are satisfied:

H1 $f: H \rightarrow \mathbb{R}$ is twice continuously Fréchet differentiable in $D_{0} \subset H$, where $D_{0}$ is a convex and open set.

H2 There exists an $x^{*} \in D_{0}$ such that $f^{\prime}\left(x^{*}\right)=0,\left\|f^{\prime \prime}\left(x^{*}\right)\right\| \leq \beta$, and that

$$
\left\|f^{\prime \prime}(x)-f^{\prime \prime}\left(x^{*}\right)\right\| \leq L\left\|x-x^{*}\right\|, \quad \forall x \in D_{0},
$$

where $L$ is a constant.

H3 $f^{\prime \prime}\left(x^{*}\right)$ is selfadjoint and strictly positive in the sense that $f^{\prime \prime}\left(x^{*}\right) h^{2} \geq \lambda\|h\|^{2}$, $\forall h \in H$, where $\lambda>0$, hence $f^{\prime \prime}\left(x^{*}\right)$ is invertible, $\left[f^{\prime \prime}\left(x^{*}\right)\right]^{-1} \equiv \Lambda \in \mathscr{L}\left(H^{\prime} ; H\right)$ and $\|\Lambda\| \leq \theta$.

LEMMA 2.2. Let the assumptions $\mathbf{H 1}-\mathrm{H} 3$ be true. In addition, assume that there are non-negative constants $\alpha_{1}$ and $\alpha_{2}$ such that the operator sequence $\left\{B_{k}\right\}$ defined by (2.7) satisfies

$$
\left\|B_{n+1}-\Lambda\right\|_{M} \leq\left(1+\alpha_{1} \sigma_{n}\right)\left\|B_{n}-\Lambda\right\|_{M}+\alpha_{2} \sigma_{n},
$$

where $\sigma_{n} \equiv \max \left\{\left\|x_{n}-x^{*}\right\|,\left\|x_{n+1}-x^{*}\right\|\right\}$. Then for each $\gamma \in(0,1)$ there exist $\epsilon=\epsilon(\gamma)$ and $\delta=\delta(\gamma)$, such that if $B_{0}$ and $x_{0}$ satisfy

$$
\left\|x_{0}-x^{*}\right\| \leq \epsilon, \quad\left\|B_{0}-\Lambda\right\|_{M} \leq \delta,
$$

then the sequence $\left\{x_{n}\right\}$ defined by the QNM algorithm is well-defined, converges to $x^{*}$, and satisfies

$$
\left\|x_{n+1}-x^{*}\right\| \leq \gamma\left\|x_{n}-x^{*}\right\|, \quad n=0,1, \ldots
$$

Furthermore, $B_{n}^{-1}$ exists and the sequences $\left\{\left\|B_{n}\right\|\right\}$ and $\left\{\left\|B_{n}^{-1}\right\|\right\}$ are uniformly bounded. 
PROOF. By the assumption H2 for any $\gamma \in(0,1)$ we can choose $\delta=\delta(\gamma)>0$ and $\epsilon=\epsilon(\gamma)>0$ such that

$$
\begin{gathered}
6 \beta(1+\gamma) \delta \eta_{2} \leq \gamma, \\
\left(2 \alpha_{1} \delta+\alpha_{2}\right) \epsilon /(1-\gamma) \leq \delta, \quad \epsilon<\epsilon_{0}, \\
\left(\theta+4 \eta_{2} \delta\right)\left[L \epsilon / 2+2(1+\gamma)^{2} \beta^{2} \eta_{2} \delta\right] \leq \gamma,
\end{gathered}
$$

where $\epsilon_{0}$ is so small that

$$
B\left(x^{*}, \epsilon_{0}\right) \equiv\left\{x \in H ;\left\|x-x^{*}\right\|<\epsilon_{0}\right\} \subset D_{0} .
$$

It follows from (2.11) that

$$
\left\|B_{0}-\Lambda\right\| \leq \eta_{2}\left\|B_{0}-\Lambda\right\|_{M} \leq \eta_{2} \delta
$$

and

$$
\left\|B_{0}\right\| \leq\|\Lambda\|+\left\|B_{0}-\Lambda\right\| \leq \theta+\eta_{2} \delta .
$$

Because $B_{0}=\Lambda+\left(B_{0}-\Lambda\right)$ and $\left\|B_{0}-\Lambda\right\|_{M} \leq \delta<1 / \beta \leq\left\|\Lambda^{-1}\right\|^{-1}$, by the Banach inverse theorem, we deduce that $B_{0}$ is invertible and that $\left\|A_{0}\right\|=\left\|B_{0}^{-1}\right\| \leq\left\|\Lambda^{-1}\right\| /\left(1-\left\|\Lambda^{-1}\right\|\left\|B_{0}-\Lambda\right\|\right) \leq \beta /\left(1-\beta \eta_{2} \delta\right)<\beta /\left(1-6 \beta \eta_{2} \delta\right)$. But by (2.16)

$$
1-6 \beta \eta_{2} \delta>1-\gamma /(1+\gamma)=1 /(1+\gamma)
$$

so

$$
\left\|A_{0}\right\|=\left\|B_{0}^{-1}\right\|<(1+\gamma) \beta
$$

Furthermore,

$$
\begin{aligned}
\left\|A_{0}-f^{\prime \prime}\left(x^{*}\right)\right\| & =\left\|A_{0}\left(\Lambda-B_{0}\right) f^{\prime \prime}\left(x^{*}\right)\right\| \leq\left\|A_{0}\right\|\left\|B_{0}-\Lambda\right\|\left\|f^{\prime \prime}\left(x^{*}\right)\right\| \\
& \leq(1+\gamma) \beta^{2} \eta_{2} \delta .
\end{aligned}
$$

It follows from the mean-value theorem that

$$
\begin{aligned}
\left\|x_{1}-x^{*}\right\| & =\left\|\left(x_{1}-x_{0}\right)+\left(x_{0}-x^{*}\right)\right\|=\left\|-B_{0}^{-1} f^{\prime}\left(x_{0}\right)+\left(x_{0}-x^{*}\right)\right\| \\
= & \| B_{0}^{-1}\left\{-\left[f^{\prime}\left(x_{0}\right)-f^{\prime}\left(x^{*}\right)-f^{\prime \prime}\left(x^{*}\right)\left(x_{0}-x^{*}\right)\right]\right. \\
& \left.\quad\left[A_{0}-f^{\prime \prime}\left(x^{*}\right)\right]\left(x_{0}-x^{*}\right)\right\} \| \\
\leq & \left\|B_{0}\right\|\left\{\left\|\int_{0}^{1}\left[f^{\prime \prime}\left(x^{*}+t\left(x_{0}-x^{*}\right)\right)-f^{\prime \prime}\left(x^{*}\right)\right]\left(x_{0}-x^{*}\right) d t\right\|\right. \\
& \left.+\left\|A_{0}-f^{\prime \prime}\left(x^{*}\right)\right\|\left\|x_{0}-x^{*}\right\|\right\} \\
\leq & \left(\theta+\eta_{2} \delta\right)\left[L \epsilon / 2+(1+\gamma) \beta^{2} \eta_{2} \delta\right]\left\|x_{0}-x^{*}\right\| \leq \gamma\left\|x_{0}-x^{*}\right\| .
\end{aligned}
$$


Next, from (2.13), (2.14), and (2.17) we have

$$
\left\|B_{1}-\Lambda\right\|_{M} \leq\left(1+\alpha_{1} \epsilon\right)\left\|B_{0}-\Lambda\right\|_{M}+\alpha_{2} \epsilon \leq \delta+\left(\alpha_{1} \delta+\alpha_{2}\right) \epsilon<2 d \delta .
$$

Using induction, we prove

$$
\left\|B_{k}-\Lambda\right\| \leq 2 \delta \text { and }\left\|x_{k+1}-x^{*}\right\| \leq \gamma\left\|x_{k}-x^{*}\right\| .
$$

In fact, suppose that (2.25) are true for $k \leq m-1$. By (2.13)

$$
\left\|B_{k+1}-\Lambda\right\|_{M} \leq\left(1+\alpha_{1} \epsilon \gamma^{k}\right)\left\|B_{k}-\Lambda\right\|_{M}+\alpha_{2} \epsilon \gamma^{k},
$$

that is,

$$
\left\|B_{k+1}-\Lambda\right\|_{M}-\left\|B_{k}-\Lambda\right\|_{M} \leq 2 \alpha_{1} \epsilon \gamma^{k} \delta+\alpha_{2} \epsilon \gamma^{k}=\left(2 \alpha_{1} \delta+\alpha_{2}\right) \epsilon \gamma^{k} .
$$

Adding (2.26) from $k=0$ to $m-1$, one gets

$$
\begin{aligned}
\left\|B_{m}-\Lambda\right\|_{M} & \leq\left\|B_{0}-\Lambda\right\|_{M}+\left(2 \alpha_{1} \delta+\alpha_{2}\right) \epsilon \sum_{k=0}^{m-1} \gamma^{k} \\
& <\delta+\left(2 \alpha_{1} \delta+\alpha_{2}\right) \epsilon /(1-\gamma) \leq 2 \delta .
\end{aligned}
$$

Therefore

$$
\left\|B_{m}-B_{0}\right\|_{M} \leq\left\|B_{m}-\Lambda\right\|_{M}+\left\|B_{0}-\Lambda\right\|_{M}<3 \delta \quad \forall m .
$$

In addition, by (2.11), (2.16), (2.21) and the above

$$
\left\|I-B_{0}^{-1} B_{m}\right\| \leq\left\|B_{0}^{-1}\right\|\left\|B_{m}-B_{0}\right\|<3(1+\gamma) \beta \eta_{2} \delta<\gamma<1,
$$

where $I \in \mathscr{L}\left(H^{\prime}\right)$ is the identity operator. By the Banach theorem $\left(B_{0}^{-1} B_{m}\right)^{-1}$ exists, hence $B_{m}$ is invertible. Furthermore,

$$
\begin{aligned}
\left\|A_{m}\right\| & =\left\|B_{m}^{-1}\right\|=\left\|\left[B_{0}+\left(B_{M}-B_{0}\right)\right]^{-1}\right\| \leq\left\|B_{0}^{-1}\right\| \sum\left\|B_{0}^{-1}\right\|^{k}\left\|B_{m}-B_{0}\right\|^{k} \\
& \leq(1+\gamma) \beta \sum\left[3(1+\gamma) \beta \eta_{2} \delta\right]^{k}=(1+\gamma) \beta /\left[1-3(1+\gamma) \beta \eta_{2} \delta\right] \\
& <(1+\gamma) \beta /\left(1-6 \beta \eta_{2} \delta\right)<(1+\gamma)^{2} \beta, \quad \forall m,
\end{aligned}
$$

that is, for any $m \in \mathbb{N}, B_{m}$ is invertible and $\left\{\left\|B_{m}^{-1}\right\|\right\}$ is uniformly bounded as well. Moreover,

$$
\begin{gathered}
\left\|B_{m}\right\| \leq\left\|B_{0}\right\|+\left\|B_{m}-B_{0}\right\| \leq\left(\theta+\eta_{2} \delta\right)+3 \eta_{2} \delta=\theta+4 \eta_{2} \delta, \\
\left\|A_{m}-f^{\prime \prime}\left(x^{*}\right)\right\| \\
=\left\|A_{m}\left(B_{m}-\Lambda\right) f^{\prime \prime}\left(x^{*}\right)\right\| \leq\left\|A_{m}\right\|\left\|B_{m}-\Lambda\right\|\left\|f^{\prime \prime}\left(x^{*}\right)\right\| \\
\leq(1+\gamma)^{2} \beta \eta_{2} 2 \delta \beta=2(1+\gamma)^{2} \beta^{2} \eta_{2} \delta .
\end{gathered}
$$


By the inductive assumption one has $\left\|x_{m}-x^{*}\right\|<\epsilon$ and so

$$
\begin{aligned}
& \left\|x_{m+1}-x^{*}\right\|=\left\|\left(x_{m+1}-x_{m}\right)+\left(x_{m}-x^{*}\right)\right\|=\left\|-B_{m}^{-1} f^{\prime}\left(x_{m}\right)+\left(x_{m}-x^{*}\right)\right\| \\
& =\| B_{m}^{-1}\left\{-\left[f^{\prime}\left(x_{m}\right)-f^{\prime}\left(x^{*}\right)-f^{\prime \prime}\left(x^{*}\right)\left(x_{m}-x^{*}\right)\right]\right. \\
& \left.\quad+\left[A_{m}-f^{\prime \prime}\left(x^{*}\right)\right]\left(x_{m}-x^{*}\right)\right\} \| \\
& \leq\left\|B_{m}^{-1}\right\|\left\{\left\|\int_{0}^{1}\left[f^{\prime \prime}\left(x^{*}+t\left(x_{m}-x^{*}\right)\right)-f^{\prime \prime}\left(x^{*}\right)\right]\left(x_{m}-x^{*}\right) d t\right\|\right. \\
& \left.\quad+\left\|A_{m}-f^{\prime \prime}\left(x^{*}\right)\right\|\left\|x_{m}-x^{*}\right\|\right\} \\
& \leq\left(\theta+4 \eta_{2} \delta\right)\left[L \epsilon / 2+2(1+\gamma)^{2} \beta^{2} \eta_{2} \delta\right]\left\|x_{m}-x^{*}\right\| \leq \gamma\left\|x_{m}-x^{*}\right\| .
\end{aligned}
$$

Furthermore,

$$
\left\|x_{m+1}-x^{*}\right\| \leq \gamma\left\|x_{m}-x^{*}\right\| \leq \cdots \leq \gamma^{m+1}\left\|x_{0}-x^{*}\right\|<\gamma^{m+1} \epsilon<\epsilon_{0} .
$$

Thus $\left\{x_{m}\right\} \subset D_{0}, x_{m} \rightarrow x^{*}$ in $H$, and $\left\{\left\|B_{m}\right\|\right\}$ and $\left\{\left\|B_{m}^{-1}\right\|\right\}$ are uniformly bounded.

LEMMA 2.3. Let $C, B \in \mathscr{L}\left(H^{\prime} ; H\right)$ be selfadjoint, $y \in H^{\prime}, s \in H$ with $\langle y, s\rangle \neq 0$, and set

$$
\bar{B}=B+\frac{(s-B y)\langle\cdot, s\rangle+s\langle\cdot, s-B y\rangle}{\langle y, s\rangle}-\frac{\langle y, s-B y\rangle}{\langle y, s\rangle^{2}} s\langle\cdot, s\rangle
$$

If $M \in \mathscr{L}(H)$ is invertible and selfadjoint, then

$$
\bar{E}=P^{*} E P+\frac{K M(s-C y)}{\langle y, s\rangle}\langle K M s, \cdot\rangle+\frac{K M s}{\langle y, s\rangle}\left\langle P^{*} K M(s-C y), \cdot\right\rangle,
$$

where $E=K M(B-C) K M, \bar{E}=K M(\bar{B}-C) K M, P=I-$ $M^{-1} K^{-1} y\langle K M s, \cdot\rangle /\langle y, s\rangle, I \in \mathscr{L}(H)$ is the identity operator, and $P^{*}$ is the adjoint operator of $P$.

ProOF. Premultiplying and postmultiplying both sides of (2.34) by $K M$ and subtract- 
ing $K M C K M$, we have the simple calculation

$$
\begin{aligned}
\bar{E}=E & +\{K M[(s-C y)-(B-C) y]\langle K M s, \cdot\rangle+K M s\langle K M[(s-C y) \\
& -(B-C) y], \cdot\rangle\} /\langle y, s\rangle-K M s\langle y,(s-C y)-(B-C) y\rangle\langle K M s, \cdot\rangle /\langle y, s\rangle^{2} \\
=E & -E M^{-1} K^{-1} y\langle K M s, \cdot\rangle /\langle y, s\rangle-K M s\left\langle E M^{-1} K^{-1} y, \cdot\right\rangle /\langle y, s\rangle \\
& +\{K M(s-C y)\langle K M s, \cdot\rangle+K M s\langle K M(s-C y), \cdot\rangle\} /\langle y, s\rangle \\
& +\left\langle E M^{-1} K^{-1} y, M^{-1} K^{-1} y\right\rangle K M s\langle K M s, \cdot\rangle /\langle y, s\rangle^{2} \\
& -\langle y, s-C y\rangle K M s\langle K M s, \cdot\rangle /\langle y, s\rangle^{2} \\
=E & {\left[I-M^{-1} K^{-1} y\langle K M s, \cdot\rangle /\langle y, s\rangle\right]+K M s\left\{\left\langle E M^{-1} K^{-1} y, M^{-1} y\right\rangle\langle K M s, \cdot\rangle /\langle y, s\rangle\right.} \\
& -\left\langle E M^{-1} K^{-1} y, \cdot \cdot\right\} /\langle y, s\rangle+K M(s-C y)\langle K M s, \cdot\rangle /\langle y, s\rangle \\
& +K M s\{\langle K M(s-C y), \cdot\rangle-\langle y, s-C y\rangle\langle K M s, \cdot\rangle /\langle y, s\rangle\} /\langle y, s\rangle .
\end{aligned}
$$

It is obvious that $E$ and $\bar{E}$ are selfadjoint and that

$$
P^{*}=I-K M s\left\langle\cdot, M^{-1} K^{-1} y\right\rangle /\langle y, s\rangle \in \mathscr{L}\left(H^{\prime}\right) .
$$

Considering the above results, from (2.36) we have

$$
\begin{aligned}
\bar{E}= & E P-K M s\left\langle E M^{-1} K^{-1} y-K M s\left\langle M^{-1} K^{-1} y, M^{-1} K^{-1} y\right\rangle /\langle y, s\rangle, \cdot\right\rangle /\langle y, s\rangle \\
& +K M(s-C y)\langle K M s, \cdot\rangle /\langle y, s\rangle+K M s\langle K M(s-C y) \\
& \left.\quad-K M s\left\langle K M(s-C y), M^{-1} K^{-1} y\right\rangle /\langle y, s\rangle, \cdot\right\rangle /\langle y, s\rangle \\
= & E P-K M s\left\langle\left[I-K M s\left\langle\cdot, M^{-1} K^{-1} y\right\rangle /\langle y, s\rangle\right] E M^{-1} K^{-1} y, \cdot\right\rangle /\langle y, s\rangle \\
& +K M(s-C y)\langle K M s, \cdot\rangle /\langle y, s\rangle \\
& +K M s\left\langle\left[I-K M s\left(\cdot, M^{-1} K^{-1} y\right\rangle /\langle y, s\rangle\right] K M(s-C y), \cdot\right\rangle /\langle y, s\rangle \\
= & E P-K M s\left\langle P^{*} E M^{-1} K^{-1} y, \cdot\right\rangle /\langle y, s\rangle+K M(s-C y)\langle K M s, \cdot\rangle /\langle y, s\rangle \\
& +K M s\left\langle P^{*} K M(s-C y), \cdot\right\rangle /\langle y, s\rangle \\
= & {\left[I-K M s\left\langle M^{-1} K^{-1} y, \cdot\right\rangle /\langle y, s\rangle\right] E P } \\
& K M(s-C y)\langle K M s, \cdot\rangle /\langle y, s\rangle+K M s\left\langle P^{*} K M(s-C y), \cdot\right\rangle /\langle y, s\rangle \\
= & P^{*} E P+K M(s-C y)\langle K M s, \cdot\rangle /\langle y, s\rangle+K M s\left\langle P^{*} K M(s-C y), \cdot\right\rangle /\langle y, s\rangle .
\end{aligned}
$$

LEMMA 2.4. Let $M \in \mathscr{L}(H)$ be a non-singular selfadjoint operator such that

$$
\left\|M s-M^{-1} K^{-1} y\right\| \leq \rho\left\|M^{-1} K^{-1} y\right\|,
$$

where $\rho \in(0,1 / 3), s \in H$ and $y \in H^{\prime}$ with $y \neq 0$. Then

$$
(1-\rho)\left\|M^{-1} K^{-1} y\right\|^{2} \leq\langle y, s\rangle \leq(1+\rho)\left\|M^{-1} K^{-1} y\right\|^{2}
$$


and for each $E \in \mathscr{B}_{2}\left(H, H^{\prime}\right)$,

$$
\begin{aligned}
& \left\|E\left[I-M^{-1} K^{-1} y\left\langle K M^{-1} K^{-1} y, \cdot\right\rangle /\langle y, s\rangle\right]\right\|_{2} \leq\left(1-\mu v^{2}\right)^{1 / 2}\|E\|_{2}, \\
& \left\|E\left[I-M^{-1} K^{-1} y\langle K M s, \cdot\rangle /\langle y, s\rangle\right]\right\|_{2} \\
& \quad \leq\left\{\left(1-\mu v^{2}\right)^{1 / 2}+\left\|M s-M^{-1} K^{-1} y\right\| /\left[(1-\rho)\left\|M^{-1} K^{-1} y\right\|\right]\right\}\|E\|_{2}
\end{aligned}
$$

and $\forall y \in H^{\prime}, A \in \mathscr{L}\left(H, H^{\prime}\right), s \in H$ :

$$
\|(y-A s)\langle K M s, \cdot\rangle /\langle y, s\rangle\|_{2} \leq 2\|y-A s\| /\left\|M^{-1} K^{-1} y\right\|,
$$

where

$$
\mu=(1-2 \rho) /\left(1-\rho^{2}\right) \in(3 / 8,1)
$$

and

$$
\nu=\left\|E M^{-1} K^{-1} y\right\| /\left(\|E\|_{2}\left\|M^{-1} K^{-1} y\right\|\right) \in(0,1) .
$$

ProOf. We have (2.39) from

$$
\begin{aligned}
\langle y, s\rangle & =\left(K^{-1} y, s\right)=\left(M M^{-1} K^{-1} y, s\right)=\left(M^{-1} K^{-1} y, M s-M^{-1} K^{-1} y+M^{-1} K^{-1} y\right) \\
& =\left\|M^{-1} K^{-1} y\right\|^{2}+\left(M^{-1} K^{-1} y, M s-M^{-1} K^{-1} y\right) .
\end{aligned}
$$

Observing that $\forall u, v \in H$,

$$
\begin{aligned}
\| E[I & -u(v, \cdot)] \|_{2}^{2} \\
& =\sum_{k}\left\|[E-E u(v, \cdot)] \phi_{k}\right\|^{2} \\
& =\sum_{k}\left(\left(E \phi_{k}-\left(v, \phi_{k}\right) E u, E \phi_{k}-\left(v, \phi_{k}\right) E u\right)\right) \\
& =\sum_{k}\left(\left(E \phi_{k}, E \phi_{k}\right)\right)-2 \sum_{k}\left(v, \phi_{k}\right)\left(\left(E u, E \phi_{k}\right)\right)+\sum_{k}\left(v, \phi_{k}\right)^{2}((E u, E u)) \\
& =\|E\|_{2}^{2}-2\left(\left(E u, E \sum_{k}\left(v, \phi_{k}\right) \phi_{k}\right)\right)+\|v\|^{2}\|E u\|^{2} \\
& =\|E\|_{2}^{2}-2((E u, E v))+\|E u\|^{2}\|v\|^{2},
\end{aligned}
$$

and taking $u=M^{-1} K^{-1} y /\langle y, s\rangle$ and $v=M^{-1} K^{-1} y$, we immediately obtain

$$
\begin{aligned}
\| E[I- & \left.M^{-1} K^{-1} y\left(M^{-1} K^{-1} y, \cdot\right) /\langle y, s\rangle\right] \|_{2}^{2} \\
= & \|E\|_{2}^{2}-2\left(E M^{-1} K^{-1} y, E M^{-1} K^{-1} y\right) /\langle y, s\rangle \\
& \quad+\left\|E M^{-1} K^{-1} y\right\|^{2}\left\|M^{-1} K^{-1} y\right\|^{2} /\langle y, s\rangle^{2} \\
= & \|E\|_{2}^{2}+\left\{-2(y, s\rangle+\left\|M^{-1} K^{-1} y\right\|^{2}\right\}\left\|E M^{-1} K^{-1} y\right\|^{2} /\langle y, s\rangle^{2} \\
\leq & \|E\|_{2}^{2}-(1-2 \rho)\left\|E M^{-1} K^{-1} y\right\|^{2} /\left[(1-\rho)\left\|M^{-1} K^{-1} y\right\|^{2}\right]=\left(1-\mu v^{2}\right)\|E\|_{2}^{2},
\end{aligned}
$$


which reduces to (2.40).

Owing to (2.40), in order to establish (2.41) we need only prove

$$
\begin{aligned}
&\left\|E M^{-1} K^{-1} y\left[\langle K M s, \cdot\rangle-\left(M^{-1} K^{-1} y, \cdot\right)\right] /\langle y, s\rangle\right\|_{2} \\
&=\left\|E M^{-1} K^{-1} y\left(M^{-1} K^{-1} y-M s, \cdot\right) /\langle y, s\rangle\right\|_{2} \\
&=\left\|M^{-1} K^{-1} y\right\|\left\|M^{-1} K^{-1} y-M s\right\| /\langle y, s\rangle\|\| E \|_{2} \\
& \leq\left\|M^{-1} K^{-1} y-M s\right\| /\left[(1-\rho)\left\|M^{-1} K^{-1} y\right\|\right]\|E\|_{2} .
\end{aligned}
$$

Inequality (2.42) can be reduced in a similar fashion. In fact,

$$
\begin{aligned}
& \|(y-A s)\langle K M s, \cdot) /\langle y, s\rangle\|_{2}^{2}=\sum_{k}\left\|(y-A s)\left(M s, \phi_{k}\right) /\langle y, s\rangle\right\|^{2} \\
& =\sum_{k}\|y-A s\|^{2}\left(M s, \phi_{k}\right)^{2} /\langle y, s\rangle^{2}=\|y-A s\|^{2}\|M s\|^{2} /\langle y, s\rangle^{2},
\end{aligned}
$$

so

$$
\begin{aligned}
\|(y- & A s)\langle K M s, \cdot\rangle /\langle y, s\rangle\left\|_{2}=\right\| y-A s\|\| M s \| /\langle y, s\rangle \\
& \leq\|y-A s\| /\langle y, s\rangle\left\{\left\|M^{-1} K^{-1} y-M s\right\|+\left\|M^{-1} K^{-1} y\right\|\right\} \\
& \leq\|y-A s\| /\left[(1-\rho)\left\|M^{-1} K^{-1} y\right\|^{2}\right](1+\rho)\left\|M^{-1} K^{-1} y\right\| \\
& \leq 2\|y-A s\| /\left\|M^{-1} K^{-1} y\right\| .
\end{aligned}
$$

LEMMA 2.5. Let $M$ satisfy the conditions in Lemma 2.4 and let $B_{0}-\Lambda \in \mathscr{B}_{2}(H)$. Then $\left\langle s_{k}, y_{k}\right\rangle \neq 0$, and $B_{k+1}$ is well-defined and satisfies

$$
\begin{aligned}
& \left\|B_{k+1}-\Lambda\right\|_{M} \leq 2(1+\rho)\|K M\|\left\|s_{k}-\Lambda y_{k}\right\| /\left[(1-\rho)^{2}\left\|M^{-1} K^{-1} y_{k}\right\|\right] \\
& \quad+\left\{\sqrt{1-\mu \nu^{2}}+5 / 2\left\|M s_{k}-M^{-1} K^{-1} y_{k}\right\| /\left[(1-\rho)\left\|M^{-1} K^{-1} y_{k}\right\|\right]\right\}\left\|B_{k}-\Lambda\right\|_{M},
\end{aligned}
$$

where $\mu=(1-2 \rho) /\left(1-\rho^{2}\right) \in[3 / 8,1]$ and $v=\left\|K M\left(B_{k}-\Lambda\right) y_{k}\right\| /\left(\| B_{k}-\right.$ $\left.\Lambda\left\|_{M}\right\| M^{-1} K^{-1} y_{k} \|\right)$.

PROOF. It follows by the QNM algorithm and (2.39) that $y_{k} \neq 0$ and $\left\langle s_{k}, y_{k}\right\rangle \neq 0$. So $B_{k+1}$ is well-defined by (2.7).

According to Lemma 2.3 we have

$$
\begin{gathered}
E_{k+1}=P^{*} E_{k} P+K M\left(s_{k}-\Lambda y_{k}\right)\left\langle K M s_{k}, \cdot\right\rangle /\left\langle y_{k}, s_{k}\right\rangle \\
+M s_{k}\left\langle P^{*} K M\left(s_{k}-\Lambda y_{k}\right), \cdot\right\rangle /\left\langle y_{k}, s_{k}\right\rangle,
\end{gathered}
$$

where $E_{k+1}=K M\left(B_{k+1}-\Lambda\right) K M, E_{k}=K M\left(B_{k}-\Lambda\right) K M$, and $P=I-$ $M^{-1} K^{-1} y_{k}\left\langle K M s_{k}, \cdot\right\rangle /\left\langle y_{k}, s_{k}\right\rangle$. 
Applying Lemma 2.4, we have

$\left\|P^{*} E_{k} P\right\|_{2} \leq\left\{\sqrt{1-\mu \nu^{2}}+\left\|M s_{k}-M^{-1} K^{-1} y_{k}\right\| /\left[(1-\rho)\left\|M^{-1} K^{-1} y_{k}\right\|\right]\right\}\left\|P^{*} E_{k}\right\|_{2}$ $\leq\left\{1+\left\|M s_{k}-M^{-1} K^{-1} y_{k}\right\| /\left[(1-\rho)\left\|M^{-1} K^{-1} y_{k}\right\|\right]\right\}\left\|E_{k} P\right\|_{2}$.

Hence,

$$
\begin{aligned}
\left\|P^{*} E P\right\|_{2} & \leq\left\{1+\left\|M s_{k}-M^{-1} K^{-1} y_{k}\right\| /\left[(1-\rho)\left\|M^{-1} K^{-1} y_{k}\right\|\right]\right\} \\
& \times\left\{\sqrt{1-\mu \nu^{2}}+\left\|M s_{k}-M^{-1} K^{-1} y_{k}\right\| /\left[(1-\rho)\left\|M^{-1} K^{-1} y_{k}\right\|\right]\right\}\left\|E_{k}\right\|_{2} \\
\leq & \left\{\sqrt{1-\mu \nu^{2}}+5\left\|M s_{k}-M^{-1} K^{-1} y_{k}\right\| /\left[2(1-\rho)\left\|M^{-1} K^{-1} y_{k}\right\|\right]\right\}\left\|E_{k}\right\|_{2}
\end{aligned}
$$

Next, we estimate the other two terms of (2.45). Since

$$
\begin{aligned}
& \left\|K M\left(s_{k}-\Lambda y_{k}\right)\left\langle K M s_{k}, \cdot\right\rangle /\left\langle y_{k}, s_{k}\right\rangle\right\|_{2}^{2}=\sum_{n}\left\|\left(M s_{k}, \phi_{n}\right) K M\left(s_{k}-\Lambda y_{k}\right)\right\|^{2} /\left\langle y_{k}, s_{k}\right)^{2} \\
& \quad=\sum_{n}\left(M s_{k}, \phi_{n}\right)^{2}\left\|K M\left(s_{k}-\Lambda y_{k}\right)\right\|^{2} /\left\langle y_{k}, s_{k}\right\rangle^{2} \\
& \quad=\left\|M s_{k}\right\|^{2}\left\|K M\left(s_{k}-\Lambda y_{k}\right)\right\|^{2} /\left\langle y_{k}, s_{k}\right\rangle^{2}
\end{aligned}
$$

and

$$
\left\|M s_{k}\right\| \leq\left\|M^{-1} K^{-1} y_{k}\right\|+\left\|M s_{k}-M^{-1} K^{-1} y_{k}\right\| \leq(1+\rho)\left\|M^{-1} K^{-1} y_{k}\right\|,
$$

we have

$$
\begin{aligned}
& \left\|K M\left(s_{k}-\Lambda y_{k}\right)\left\langle K M s_{k}, \cdot\right\rangle /\left\langle y_{k}, s_{k}\right)\right\|_{2}=\left\|M s_{k}\right\|\left\|K M\left(s_{k}-\Lambda y_{k}\right)\right\| /\left\langle y_{k}, s_{k}\right\rangle \\
& \quad \leq(1+\rho)\left\|M^{-1} K^{-1} y_{k}\right\|\|K M\|\left\|s_{k}-\Lambda y_{k}\right\| /\left[(1-\rho)\left\|M^{-1} K^{-1} y_{k}\right\|^{2}\right] \\
& \quad=(1+\rho)\|K M\|\left\|s_{k}-\Lambda y_{k}\right\| /\left[(1-\rho)\left\|M^{-1} K^{-1} y_{k}\right\|\right] .
\end{aligned}
$$

On the other hand,

$$
\begin{aligned}
\left\|P^{*}\right\| & =\|P\| \leq\|I\|+\left\|M^{-1} K^{-1} y_{k}\left\langle K M s_{k}, \cdot\right\rangle /\left\langle y_{k}, s_{k}\right\rangle\right\| \\
& \leq 1+(1+\rho) /(1-\rho)=2 /(1-\rho),
\end{aligned}
$$

and hence

$$
\begin{aligned}
\| K M & s_{k}\left(P^{*} K M\left(s_{k}-\Lambda y_{k}\right), \cdot\right) /\left\langle y_{k}, s_{k}\right\rangle \|_{2} \\
& =\left\|P^{*} K M\left(s_{k}-\Lambda y_{k}\right)\right\|\left\|M s_{k}\right\| /\left\langle y_{k}, s_{k}\right\rangle \\
& \leq\left\|P^{*}\right\|\|K M\|\left\|s_{k}-\Lambda y_{k}\right\|(1+\rho)\left\|M^{-1} K^{-1} y_{k}\right\| /\left[(1-\rho)\left\|M^{-1} K^{-1} y_{k}\right\|^{2}\right] \\
& \leq 2(1+\rho)\|K M\|\left\|s_{k}-\Lambda y_{k}\right\| /\left[(1-\rho)\left\|M^{-1} K^{-1} y_{k}\right\|\right] .
\end{aligned}
$$


Summing up (2.46), (2.47) and (2.49) and considering $\left\|B_{k+1}-\Lambda\right\|_{M}=$ $\left\|K M\left(B_{k+1}-\Lambda\right) K M\right\|_{2}=\left\|E_{k+1}\right\|_{2}$, we immediately obtain the estimate (2.44) from (2.45).

THEOREM 2.6. Let assumptions $\mathrm{H1}, \mathrm{H} 2$, and $\mathrm{H3}$ be satisfied. Then the sequence $\left\{x_{n}\right\}$ generated by the QNM algorithm is well-defined and converges to $x^{*}$ provided that the initial guesses $x_{0}$ and $B_{0}$ satisfy the conditions of Lemma 2.2 .

Proof. Because $f^{\prime \prime}\left(x^{*}\right)[\cdot, \cdot]$ is a bounded symmetric bilinear form on $H \times H$, there is a selfadjoint operator $T \in \mathscr{L}(H)$ such that

$$
f^{\prime \prime}\left(x^{*}\right)[s, t]=(T s, t), \quad \forall s, t \in H .
$$

Moreover, by assumption $\mathbf{H 3}$, there exists a selfadjoint positive operator $M \in \mathscr{L}(H)$ such that $M^{2}=T$. Hence

$$
f^{\prime \prime}\left(x^{*}\right)[s, t]=\left(M^{2} s, t\right)=(M s, M t), \quad \forall s, t \in H .
$$

For any $y \in H^{\prime}$ we have $K^{-1} y \in H$ and

$$
y-f^{\prime \prime}\left(x^{*}\right) s(\cdot)=\left(K^{-1} y-M^{2} s, \cdot\right)=K\left[M\left(M^{-1} K^{-1} y-M s\right)\right](\cdot) .
$$

But, by assumption $\mathbf{H} 2$ and the QNM algorithm one has

$$
\begin{aligned}
\| y_{k}- & f^{\prime \prime}\left(x^{*}\right) s_{k}\|=\| f^{\prime}\left(x_{k+1}\right)-f^{\prime}\left(x_{k}\right)-f^{\prime \prime}\left(x^{*}\right) s_{k} \| \\
& =\| \int_{0}^{1}\left[f^{\prime \prime}\left(x^{*}+t\left(x_{k+1}-x_{k}\right)-f^{\prime \prime}\left(x^{*}\right)\right]\left(x_{k+1}-x_{k}\right) d t \|\right. \\
& \leq L\left\|x_{k+1}-x_{k}\right\|^{2} / 2=L\left\|s_{k}\right\|^{2} / 2 .
\end{aligned}
$$

So

$$
\begin{aligned}
\| M s_{k} & -M^{-1} K^{-1} y_{k}\|=\| M^{-1} K^{-1}\left[y_{k}-f^{\prime \prime}\left(x^{*}\right) s_{k}\right] \| \\
& \leq\left\|M^{-1} K^{-1}\right\| L\left\|s_{k}\right\|^{2} / 2 .
\end{aligned}
$$

Moreover, by assumption $\mathrm{H3}$ there is a $\kappa>0$ such that

$$
\left\|s_{k}\right\| / \kappa \leq\left\|M^{-1} K^{-1} y_{k}\right\| \leq \kappa\left\|s_{k}\right\| .
$$

Summarizing (2.50), (2.51) and (2.53), we have

$$
\left\|M s_{k}-M^{-1} y_{k}\right\| \leq \rho\left\|M^{-1} K^{-1} y_{k}\right\|,
$$


where $\rho \in(0,1 / 3)$. It follows by Lemma 2.1 that $B_{k}-\Lambda \in \mathscr{B}_{2}\left(H^{\prime}, H\right)$. Therefore by Lemma 2.5

$$
\begin{aligned}
\| B_{k+1} & -\Lambda \|_{M} \leq\left\{\sqrt{1-\mu \nu^{2}}+5\left\|M s_{k}-M^{-1} K^{-1} y_{k}\right\| /\left[2(1-\rho)\left\|M^{-1} K^{-1} y_{k}\right\|\right]\right\} \\
& \times\left\|B_{k}-\Lambda\right\|_{M}+2(1+\rho)\|K M\|\left\|s_{k}-\Lambda y_{k}\right\| /\left[(1-\rho)^{2}\left\|M^{-1} K^{-1} y_{k}\right\|\right] .
\end{aligned}
$$

Considering (2.52) and (2.53), we have

$$
\begin{aligned}
\left\|s_{k}-\Lambda y_{k}\right\| & =\| \Lambda\left(y_{k}-f^{\prime \prime}\left(x^{*}\right) s_{k}\|\leq\| \Lambda\|L\| s_{k} \|^{2} / 2\right. \\
& \leq \theta L \kappa\left\|M^{-1} K^{-1} y_{k}\right\| / 2\left\|s_{k}\right\| .
\end{aligned}
$$

If we set $\alpha_{1}=5 L \kappa \| M^{-1} K^{-1} /[4(1-\rho)]$ and $\alpha_{2}=(1+\rho) L \kappa\|K M\| /(1-\rho)^{2}$, then from (2.55) we have

$$
\left\|B_{k+1}-\Lambda\right\|_{M} \leq\left(1+\alpha_{1} \sigma_{k}\right)\left\|B_{k}-\Lambda\right\|_{M}+\alpha_{2} \sigma_{k},
$$

where $\sigma_{k} \equiv \max \left(\left\|x_{k+1}-x^{*}\right\|,\left\|x_{k}-x^{*}\right\|\right)$.

It follows by Lemma 2.2 that the conclusions of Theorem 2.6 are true.

From [8] we quote the following result.

THEOREM 2.7. Assume that the requirements in Theorem 2.6 are satisfied and that $A_{0}-F^{\prime}\left(q^{*}\right)$ is compact. Then the sequence $\left\{x_{n}\right\}$ generated by $\mathrm{QNM}$ is $\mathrm{Q}$-superlinear convergent, that is,

$$
\lim _{k \rightarrow \infty}\left\|x_{k+1}-x^{*}\right\| /\left\|x_{k}-x^{*}\right\|=0,
$$

provided $\left\|x_{0}-x^{*}\right\|$ is sufficiently small.

\section{Identification of a nonlinear parabolic system}

The problem we address here is to identify the parameter $q$ appearing in a parabolic semilinear equation

$$
\begin{aligned}
\partial_{t} u-\Delta u+q^{2} u^{2} & =0, & & (x, t) \in D \equiv \Omega \times(0, T), \\
\left.u\right|_{\partial \Omega} & =0, & & \left.u\right|_{t=0}=g(x),
\end{aligned}
$$

based on the final measurement of the state $u$

$$
\left.u\right|_{t=T}=z(x), \quad x \in \Omega .
$$

The assumptions we use in this section are as follows: 
A1. $\Omega \subset \mathbb{R}^{m}$ is bounded and its boundary $\partial \Omega \in C^{2+\beta}$, where $\beta \in(0,1)$;

A2. $g \in C^{2+\beta}(\bar{\Omega})$.

For any $q \in C^{\beta}(\bar{\Omega})$ it was proved in [14] that problem (3.1) has a unique classical solution, $u \in C^{2+\beta .1+\beta / 2}(\bar{D})$. So, we denote $u=u(q)=u(x, t ; q)$ to show the dependence of $u$ on $q$.

The problem of identification is stated as an optimization problem

$$
I(q)=1 / 2\|u(\cdot, T ; q)-z\|_{L^{2}(\Omega)}^{2} \rightarrow \min .
$$

But the above problem is, usually, ill-posed in the Hadamard sense. Thus, we introduce a regularization term as follows:

$$
J(q)=1 / 2\|u(\cdot, T ; q)-z\|_{L^{2}(\Omega)}^{2}+\alpha / 2\|q\|_{H}^{2},
$$

where $\alpha>0$ is a constant, $H=H^{l}(\Omega)$, and the order $l$ of the Sobolev space is chosen such that $H$ is compactly embedded in $C^{\beta}(\bar{\Omega})$. For example, $l=1$ when $m=1$ and $l=2$ when $m=2$ or 3 .

It follows by [21] that the optimal parameter for the problem (3.4) converges to the optimal parameter of the problem (3.3) as $\alpha \rightarrow 0$.

THEOREM 3.1. The function $u: H \rightarrow V \equiv C^{2+\beta .1+\beta / 2}(\bar{D})$ defined by (3.1) is infinitely differentiable, that is, $u \in C^{N}(H ; V), \forall N \in \mathbb{N} \cup\{0\}$, where $C^{N}(H ; V)$ denotes the linear space of $N$-times continuously Fréchet differentiable functions on $H$ to $V$. Moreover, the first Fréchet derivative $u^{\prime}(\cdot): H \rightarrow \mathscr{L}(H ; V)$ and the second Fréchet derivative $u^{\prime \prime}(\cdot): H \rightarrow \mathscr{L}(H ; \mathscr{L}(H ; V))$ of $u$ at $q$ are implicitly determined by $u^{\prime}(q) h=\dot{u}$ and $u^{\prime \prime}(q) h k=\ddot{u}, \forall h, k \in H$, respectively, where $\dot{u}$ and $\ddot{u}$ are determined by the problems

$$
\begin{gathered}
\partial_{t} \dot{u}-\Delta \dot{u}+2 q^{2} u \dot{u}=-2 q u^{2} h, \quad(x, t) \in D \\
\left.\dot{u}\right|_{\partial \Omega}=0,\left.\quad \dot{u}\right|_{t=0}=0,
\end{gathered}
$$

and

$$
\begin{gathered}
\partial_{t} \ddot{u}-\Delta \ddot{u}+2 q^{2} u \ddot{u}=-4 q u \dot{v} h-4 q u \dot{u} k-2 q^{2} \dot{u} \dot{v}-2 u^{2} h k, \quad(x, t) \in D \\
\left.\ddot{u}\right|_{\partial \Omega}=0,\left.\quad \ddot{u}\right|_{t=0}=0,
\end{gathered}
$$

where $u=u(q)$ is determined by (3.1), $\dot{u}=u^{\prime}(q) h$ and $\dot{v}=u^{\prime}(q) k$ are determined by (3.5), respectively.

Proof. First, we prove $u \in C^{N}(H ; V)$. 
Take, for example, $N=0$. The proof is similar for any order $N$. Let $q, \bar{q} \in H$. Then it follows from (3.1) that $\bar{u}=u(\bar{q})$ and $u=u(q)$. Set $h=\bar{q}-q$ and $\delta u=\bar{u}-u$, so $\delta u$ satisfies

$$
\begin{gathered}
\partial_{t}(\delta u)-\Delta(\delta u)+q^{2}(\bar{u}+u)(\delta u)=-(\bar{q}+q) \bar{u}^{2} h, \quad(x, t) \in D, \\
\left.(\delta u)\right|_{\partial \Omega}=0,\left.\quad(\delta u)\right|_{t=0}=0 .
\end{gathered}
$$

It is obvious by [14] that $\|\delta u\|_{V}=O\left(\|h\|_{H}\right)$. Hence $u(\cdot) \in C(H ; V)$.

Secondly, we prove $u^{\prime}(q) h=\dot{u}$. For $q, h \in H$, there exists a unique solution $\dot{u} \in V$ to the problem (3.5). Thus, set $\bar{q}=q+h, u=u(q), \bar{u}=u(\bar{q})$, and $\tilde{u}=\bar{u}-u-\dot{u}=\delta u-\dot{u}$. It is evident that $\tilde{u}$ satisfies

$$
\begin{gathered}
\partial_{t} \tilde{u}-\Delta \tilde{u}+q^{2}(\bar{u}+u) \tilde{u}=-q^{2} \dot{u} \delta u-(\bar{q}+q)(\bar{u}+u) h \delta u-u^{2} h^{2}, \quad(x, t) \in D, \\
\left.\tilde{u}\right|_{\partial \Omega}=0,\left.\quad \tilde{u}\right|_{t=0}=0 .
\end{gathered}
$$

It follows from (3.5) by [14] that $\dot{u}=O\left(\|h\|_{H}\right)$. Moreover, by the above argument we obtain $\delta u=o(1)$. Therefore, $\tilde{u}=o(\|h\|)$. Hence, $u^{\prime}(q) h=\dot{u}$. It is similar to prove $u^{\prime \prime}(q) h k=\ddot{u}$.

Next, we give the following result.

THEOREM 3.2. There exists an optimal element for the optimization problem (3.4).

PROOF. Suppose that $\left\{q_{n}\right\}$ is a minimizing sequence for the optimization problem (3.4), that is,

$$
J\left(q_{n}\right) \rightarrow h \equiv \inf _{q \in H} J(q) .
$$

Thus $\left\{q_{n}\right\}$ is bounded in $H$. Since $H$ is a Hilbert space, there exists a subsequence, which is still denoted $\left\{q_{n}\right\}$, such that $q_{n} \stackrel{w}{\rightarrow} \bar{q}$, in $H .^{1}$ By the property of compact embeddedness of $H$, it follows that $q_{n} \stackrel{s}{\rightarrow} \bar{q}$ in $C^{\beta}(\bar{\Omega})$. Furthermore, by Theorem 3.1 we obtain that $u\left(q_{n}\right) \stackrel{s}{\rightarrow} u(\bar{q})$ in $C^{2+\beta, 1+\beta / 2}(\bar{D})$.

We can write $J\left(q_{n}\right)$ as

$$
J\left(q_{n}\right)=1 / 2\left\|u\left(\cdot, T ; q_{n}\right)-z\right\|_{L^{2}(\Omega)}^{2}+\alpha / 2\left\|q_{n}\right\|_{H}^{2} \equiv J_{1}\left(q_{n}\right)+J_{2}\left(q_{n}\right) .
$$

Obviously, $J_{2}\left(q_{n}\right) \equiv \alpha / 2\left\|q_{n}\right\|_{H}^{2}$ is convex and strongly lower semi-continuous, so it is also weakly lower semi-continuous. Letting $n \rightarrow \infty$ in (3.9) and considering (3.8), we obtain

$$
J(\bar{q})=1 / 2\|u(\cdot, T ; \bar{q})-z\|_{L^{2}(\Omega)}^{2}+\alpha / 2\|\bar{q}\|_{H}^{2}=\inf _{q \in H} J(q) .
$$

That is, $\bar{q}$ is the optimal element.

\footnotetext{
${ }^{1 "} x_{n} \stackrel{s}{\rightarrow} \bar{x}$ (or $x_{n} \stackrel{w}{\rightarrow} \bar{x}$ ), in $X$ " means that $x_{n}$ strongly (or weakly) converges to $\bar{x}$ in $X$.
} 
For calculating $J^{\prime}(q)$ we have the following results.

THEOREM 3.3. The functional $J(q)$ defined by (3.4) is twice continuously Fréchet differentiable and its first Fréchet differential $J^{\prime}(q) h$ is determined by the formula

$$
J^{\prime}(q) h=\langle\tilde{j}(q), h\rangle, \quad \forall h \in H,
$$

where $\tilde{j}(q) \in H^{\prime}$ is defined by

$$
\tilde{j}(q) \equiv-2 \int_{0}^{T} q p(q) u^{2}(q) d t+\alpha K q,
$$

$K: H \rightarrow H^{\prime}$ is the canonical isometry, $u(q)=u$ is determined by (3.1), and $p(q)=p$ is defined by the problem

$$
\begin{gathered}
-\partial_{t} p-\Delta p+2 q^{2} u p=0, \quad(x, t) \in D, \\
\left.p\right|_{\partial \Omega}=0,\left.\quad p\right|_{t=T}=u(T ; q)-z .
\end{gathered}
$$

Moreover, the second Fréchet differential $J^{\prime \prime}(q) h k$ is determined by the formula

$$
\begin{gathered}
J^{\prime \prime}(q) h k=\int_{0}^{T}\left\langle-4 q u \dot{u} k-4 q u \dot{v} h-2 q^{2} \dot{u} \dot{v}-2 u^{2} h k, p\right\rangle d t \\
+\langle\dot{u}(T), \dot{v}(T)\rangle+\alpha(h, k), \quad \forall h, k \in H,
\end{gathered}
$$

where $\dot{u}=u^{\prime}(q) h$ and $\dot{v}=u^{\prime}(q) k$ are determined by $(3.5)$ and $\langle\cdot, \cdot\rangle$ and $(\cdot, \cdot)$ denote the inner products in $L^{2}(\Omega)$ and $H$, respectively.

PROOF. It follows from the calculation

$$
\begin{aligned}
J(q+h) & -J(q)-\{\langle\dot{u}(T ; q), u(T ; q)-z\rangle+\alpha(q, h)\} \\
= & 1 / 2\left\langle u(T ; q+h)-u(T ; q)-u^{\prime}(q) h(T), u(T ; q+h)-z\right\rangle \\
& +1 / 2\left\langle u^{\prime}(q) h(T), u(T ; q+h)-u(T ; q)\right\rangle \\
& +1 / 2\left\langle u(T ; q)-z, u(T ; q+h)-u(T ; q)-u^{\prime}(q) h(T)\right\rangle+\alpha / 2\|h\|_{H}^{2} \\
= & o(\|h\|),
\end{aligned}
$$

that

$$
J^{\prime}(q) h=\langle\dot{u}(T ; q), u(T ; q)-z\rangle+\alpha(q, h), \quad \forall h \in H .
$$


Consideration of $\dot{u}(0 ; q)=0$ and $p(T ; q)=u(T ; q)-z$ and use of Green's formula lead to

$$
\begin{aligned}
& \langle\dot{u}(T ; q), u(T ; q)-z\rangle=\langle\dot{u}(T ; q), p(T ; q)\rangle=\int_{0}^{T} \partial_{t}\langle\dot{u}, p\rangle d t \\
& \quad=\int_{0}^{T}\left\{\left\langle\partial_{t} \dot{u}, p\right\rangle+\left\langle\dot{u}, \partial_{t} p\right\rangle\right\} d t=\int_{0}^{T}\left\{\left\langle\partial_{t} \dot{u}, p\right\rangle+\left\langle\dot{u},-\Delta p+2 q^{2} u p\right\rangle\right\} d t \\
& \quad=\int_{0}^{T}\left\langle\partial_{t} \dot{u}-\Delta \dot{u}+2 q^{2} u \dot{u}, p\right\rangle d t=\left\langle-\int_{0}^{T} 2 q u^{2} p d t, h\right\rangle
\end{aligned}
$$

Since $H$ is a Sobolev space of order $l$ with $l>0, H$ is compactly embedded in $L^{2}(\Omega)$. If we choose $L^{2}(\Omega)$ to be a pivot space, that is , $L^{2}(\Omega)=\left[L^{2}(\Omega)\right]^{\prime}$, then

$$
H \subset L^{2}(\Omega) \subset H^{\prime}
$$

Because $K$ is the canonical isometry from $H$ onto $H^{\prime}$,

$$
(q, h)=\langle K q, h\rangle_{L^{2}(\Omega)}, \quad \forall q, h \in H .
$$

Substitution of (3.15) and (3.17) into (3.14) leads to

$$
J^{\prime}(q) h=\left\langle-2 \int_{0}^{T} q u^{2} p d t+K q, h\right\rangle, \quad \forall h \in H .
$$

From (3.11) we see that (3.10) holds.

Furthermore, by (3.14) we obtain

$$
\begin{aligned}
& J^{\prime}(q+k) h-J^{\prime}(q) h-\left\{\left\langle u^{\prime \prime}(q) h k(T), u(T ; q)-z\right\rangle+\langle\dot{u}(T), \dot{v}(T)\rangle+\alpha(h, k)\right\} \\
&=\left\{\left\langle u^{\prime}(q+k) h(T), u(T ; q+k)-z\right\rangle+\alpha(q+k, h)\right\} \\
&-\left\{\left\langle u^{\prime}(q) h(T), u(T ; q)-z\right\rangle+\alpha(q, h)\right\} \\
&-\{\langle\ddot{u}(T), u(T ; q)-z\rangle+\langle\dot{u}(T), \dot{v}(T)\rangle+\alpha(h, k)\} \\
&=\left\langle u^{\prime}(q+k) h(T)-u^{\prime}(q) h(T)-\ddot{u}(T), u(T ; q)-z\right\rangle \\
&+\left\langle u^{\prime}(q+k) h(T), u(T ; q+k)-u(T ; q)-\dot{v}(T)\right\rangle \\
&+\left\langle u^{\prime}(q+k) h(T)-u^{\prime}(q) h(T), \dot{v}(T)\right\rangle=o\left(\|h\|^{2}+\|k\|^{2}\right) .
\end{aligned}
$$

Thus, $J(q)$ is twice Fréchet differentiable and its second Fréchet differential is

$$
J^{\prime \prime}(q) h k=\langle\ddot{u}(T), u(T ; q)-z\rangle+\langle\dot{u}(T), \dot{v}(T)\rangle+\alpha(h, k) \quad \forall h, k \in H .
$$


Using $u(T ; q)-z=p(T ; q)$ from (3.12) and using an argument similar to (3.15), we have

$$
\begin{aligned}
\langle\ddot{u}(T), & u(T ; q)-z\rangle=\langle\ddot{u}(T), p(T ; q)\rangle=\int_{0}^{T} \partial_{t}\langle\ddot{u}, p\rangle d t \\
& =\int_{0}^{T}\left\{\left\langle\partial_{t} \ddot{u}, p\right\rangle+\left\langle\ddot{u},-\Delta p+2 q^{2} u p\right\rangle\right\} d t=\int_{0}^{T}\left\langle\partial_{t} \ddot{u}-\Delta \ddot{u}+2 q^{2} u \ddot{u}, p\right\rangle d t \\
& =\int_{0}^{T}\left\langle-4 q u \dot{u} k-4 q u \dot{v} h-2 q^{2} \dot{u} \dot{v}-2 u^{2} h k, p\right\rangle d t .
\end{aligned}
$$

Substituting (3.20) for the first term of (3.19) we obtain immediately (3.13).

The following Lemma is quoted from [7].

LEMMA 3.4. Let $(S, \Sigma, \mu)$ be a positive measure space. Then an operator $A$ in the Hilbert space $L^{2}(S, \Sigma, \mu)$ is of Hilbert-Schmidt class if and only if there exists a $\mu \times \mu$ measurable function $A(\cdot, \cdot)$ on $S \times S$ such that

$$
\left\{\int_{S} \int_{S}|A(x, t)|^{2} \mu(d s) \mu(d t)\right\}^{1 / 2}<\infty
$$

and such that

$$
A f(s) \equiv \int_{S} A(s, t) f(t) \mu(d t), \quad f \in L^{2}(S, \Sigma, \mu),
$$

for $\mu$-almost all s. Moreover, $\|A\|_{2}$ is exactly equal to the finite quantity (3.21).

LEMMA 3.5. The operator $I^{\prime \prime}(q): L^{2}(\Omega) \rightarrow L^{2}(\Omega)$ is of Hilbert-Schmidt class, where $I(q)$ is defined by (3.3).

PROOF. It is obvious that

$$
I^{\prime \prime}(q) h k=\left\langle u^{\prime}(T ; q) k, u^{\prime}(T ; q) h\right\rangle+\left\langle u^{\prime \prime}(q) h k(T), u(T ; q)-z\right\rangle
$$

and

$$
I^{\prime \prime}(q) h=\left[u^{\prime}(T ; q)\right]^{*} u^{\prime}(q) h(T)+\left[u^{\prime \prime}(T ; q) h\right]^{*}[u(T ; q)-z],
$$

where $\left[u^{\prime}(T ; q)\right]^{*}$ and $\left[u^{\prime \prime}(T ; q) h\right]^{*}$ are the dual operators of $u^{\prime}(T ; q)$ and $u^{\prime \prime}(T ; q) h$, respectively. Moreover, from [14] and problem (3.5), it is easy to see that

$$
u^{\prime}(T ; q) h(x)=-2 \int_{0}^{T} \int_{\Omega} G(x, \xi, T, \tau) q(\xi) u^{2}(\xi, \tau ; q) h(\xi) d \xi d \tau
$$


where $G(x, \xi, t, \tau)$ is the Green function of the problem (3.5), and hence

$$
\left[u^{\prime}(T ; q)\right]^{*} k(x)=-2 q(x) \int_{\Omega} \int_{0}^{T} G(\xi, x, T, \tau) u^{2}(x, \tau ; q) d \tau k(\xi) d \xi \quad \forall k \in H .
$$

Substitution of (3.25) into (3.26) and consideration of Lemma 3.4 lead to the conclusion that the operator

$$
\left[u^{\prime}(T ; q)\right]^{*} u^{\prime}(T ; q): L^{2}(\Omega) \rightarrow L^{2}(\Omega)
$$

is of Hilbert-Schmidt class.

We need the following assumption.

A3. Suppose that there exists a $q^{*} \in H$ such that

$$
I\left(q^{*}\right)=\min _{q \in H} I(q)
$$

It is obvious that assumption $\mathbf{A} 3$ implies $I^{\prime}\left(q^{*}\right)=0$ and $I^{\prime \prime}\left(q^{*}\right) \geq 0$. Thus,

$$
J^{\prime \prime}\left(q^{*}\right) h^{2}=I^{\prime \prime}\left(q^{*}\right) h^{2}+\alpha(h, h) \geq \alpha\|h\|_{H}^{2}, \quad \forall h \in H,
$$

that is, $J^{\prime \prime}\left(q^{*}\right)$ is strictly positive. Therefore, $q^{*}$ solves the following operator equation

$$
K^{-1} I^{\prime}\left(q^{*}\right)=0 .
$$

When $\alpha$ is small, instead of (3.29) we consider the equation

$$
f^{\prime}(q) \equiv K^{-1} J^{\prime}(q) \equiv K^{-1} I^{\prime}(q)+\alpha q=0 .
$$

Using the QNM algorithm stated in Section 2, we obtain an approximate sequence $\left\{q_{n}\right\}$, and its convergence is proved in the following theorem.

THEOREM 3.6. Let assumptions A1, A2, and $\mathbf{A} 3$ be true. The sequence $\left\{q_{n}\right\}$ determined by the QNM algorithm is superlinearly convergent providing that $q_{0}$ and $A_{0}$ satisfy the conditions of Lemma 2.2 and that $A_{0}-f^{\prime \prime}\left(q^{*}\right)$ is compact.

PROOF. Under assumptions A1-A3, the function $f^{\prime}(q)$ defined by (3.30) satisfies assumptions H1-H3 of Section 2. Thus, by Theorem 2.7 the conclusions of Theorem 3.6 follow immediately. 
In order to solve (3.30) we used the QNM algorithm for the one-dimensional case, that is, $\Omega=(0,1)$. Moreover, we take $H=H_{0}^{1}(\Omega)$, and hence $K=-\partial^{2} / \partial x^{2}$.

Suppose that $g(x)=\sin ^{2} \pi x$ and that the true parameter in (3.1) is $q_{t r}(x)=$ $x(1-x)$. Then $z$ in (3.2) is $z=u\left(T ; q_{t r}\right)$. We used the Crank-Nicholson implicit finite difference method, for example see [15], to discretize (3.1) and (3.2), that is, we could, for example, replace $\partial_{t} u(i \Delta x, j \Delta t)$ with

$$
\partial_{t} u(i \Delta x,(j+1 / 2) \Delta t) \approx 2\left(u_{i, j+1 / 2}-u_{i, j}\right) / \Delta t,
$$

where $u_{i, j}=u(i \Delta x, j \Delta t)$ and $u_{i, j+1 / 2}=u(i \Delta x,(j+1 / 2) \Delta t)$.

Therefore one could obtain the three-level stable approximate equations

$$
\frac{1}{\Delta x^{2}}\left(u_{i+1, j+1 / 2}-2 u_{i, j+1 / 2}+u_{i-1, j+1 / 2}\right)=\frac{2}{\Delta t}\left(u_{i, j+1 / 2}-u_{i, j}\right)+q_{i}^{2} u_{i, j}^{2}
$$

and

$$
\begin{aligned}
& u_{i-1, j+1} / \Delta x^{2}-2\left(1 / \Delta x^{2}+1 / \Delta t\right) u_{i, j+1}+u_{i+1, j+1} / \Delta x^{2} \\
& \quad=-u_{i-1, j} / \Delta x^{2}+2\left(1 / \Delta x^{2}-1 / \Delta t\right) u_{i, j}-u_{i+1, j} / \Delta x^{2}+q_{i}^{2} u_{i, j+1 / 2}^{2}
\end{aligned}
$$

We also have similar discrete equations for problem (3.12).

In this paper we took $T=1, \Delta x=0.1, \Delta t=0.05$, and $N_{x}=1 / \Delta x$.

The computational results are summarized in Table 1, where

$$
\begin{aligned}
\Delta q_{i} & =q(i \Delta x)-q_{t r}(i \Delta x), \\
m_{1} & =\sum_{i=0}^{N_{x}} \Delta q_{i} /\left(N_{x}+1\right), \\
\sigma_{1} & =\sqrt{\sum_{i=0}^{N_{x}}\left[\Delta q_{i}-m_{1}\right]^{2} / N_{x}}, \\
s & =\sqrt{\sum_{i=0}^{N_{x}}\left[\Delta q_{i}\right]^{2}}, \\
y_{i} & =u(i \Delta x)-z(i \Delta x) \\
m_{2} & =\sum_{i=0}^{N_{x}} y_{i} /\left(N_{x}+1\right), \\
\sigma_{2} & =\sqrt{\sum_{i=0}^{N_{x}}\left[y_{i}-m_{2}\right]^{2} / N_{x}}, \\
J & =\Delta x \sum_{i=0}^{N_{x}} y_{i}^{2} / 2+\alpha \sum_{i=0}^{N_{x}}[q((i+1) \Delta x)-q(i \Delta x)]^{2} / 2 \Delta x .
\end{aligned}
$$

\section{Acknowledgement}

This work has partially been supported by the National Nature Science Foundation of China. 
TABLE 1. Convergence for the algorithm QNM

\begin{tabular}{|c|c|c|c|c|c|c|}
\hline $\begin{array}{c}\text { Iteration } \\
\text { Times }\end{array}$ & $m_{1}$ & $\sigma_{1}$ & $s$ & $m_{2}$ & $\sigma_{2}$ & $J$ \\
\hline 1 & -8.359 & 14.087 & 53.841 & $1.25 E-5$ & $1.309 E-5$ & 635.135 \\
2 & -2.057 & 4.466 & 38.353 & $1.68 E-6$ & $1.724 E-6$ & 78.117 \\
3 & 0.849 & $9.4 E-2$ & $4.37 E-5$ & $5.21 E-7$ & $7.459 E-7$ & $1.084 E-5$ \\
4 & 0.85 & $9.06 E-2$ & $2.33 E-7$ & $3.22 E-7$ & $3.007 E-7$ & $1.381 E-13$ \\
\hline
\end{tabular}

\section{References}

[1] H. T. Banks and K. Kunisch, Estimation Techniques for Distributed Parameter Systems (Birkhäuser, Boston, 1989).

[2] H. T. Banks, S. Reich and I. G. Rosen, "An approximation theory for the identification of nonlinear distributed parameter systems", SIAM J. Control and Optim. 28 (1990) 523-569.

[3] C. G. Broyden, J. E. Dennis and J. J. Moore, "On the local and superlinear convergence of quasi-Newton methods", J. Inst. Math. Appl. 12 (1973) 223-245.

[4] G. Chavent, "Identification of distributed parameter systems: About the output least squares method, its implementation and identifiability", in: Identification and System Parameter Estimation (ed. R. Isermann) (Pergamon, New York, 1982) 85-97.

[5] Y. M. Chen and F. G. Zhang, "Hierarchical multigrid strategy for efficiency improvement of the GPST inversion algorithm", Applied Numerical Mathematics 6 (1990) 431-446.

[6] T. F. Coleman and A. R. Conn, "On the local convergence of a quasi-Newton method for the nonlinear programming problem", SIAM J. Numer. Anal. 21 (1984) 755-769.

[7] N. Dunford and J. T. Schwartz, Linear Operators, Part II: Spectral Theory (Interscience, New York, 1963).

[8] A. Griewank, "The local convergence of Broyden-like methods on Lipchitzian problems in Hilbert spaces", SIAM J. Numer. Anal. 24 (1987) 684-705.

[9] D. M. Hwang and C. T. Kelley, "Sequential quadratic programming for parameter identification problems", in: Fifih Symp. on Control of Distributed Parameter Systems, (ed. A. E. Jai and M. Amouroux) (1989) 105-109.

[10] K. Ito and K. Kunisch, "The augmented Lagrangian method for equality and inequality constrained problems in Hilbert spaces", Math. Programming 46 (1990) 341-360.

[11] K. Ito and K. Kunisch, "The augmented Lagrangian method for parameter estimation in elliptic systems", SIAM J. Control and Optim. 28 (1990) 113-136.

[12] C. T. Kelley and E. W. Sachs, "Quasi-Newton methods and unconstrained optimal control problems", SIAM J. Control and Optim. 23 (1987) 1503-1516.

[13] C. K. Kunisch and E. W. Sachs, "Reduced SQP methods for parameter identification problems", SIAM J. Numer. Anal. 29 (1992) 1793-1820.

[14] O. A. Ladyzenskaja et al., Linear and Quasi-linear Equations of Parabolic Type, (AMS, Providence, 1968).

[15] L. Lapidus and G. F. Pinder, Numerical Solution of Partial Differential Equations in Sciences and Engineering (John Wiley and Sons, New York, 1982).

[16] Tai-Yong Lee and John H. Seinfeld, "Estimation of two-phase petroleum reservoir properties by regularization", J. Comput. Phys. 69 (1987) 397-419.

[17] J. H. Seinfeld and W. H. Chen, "Identification of petroleum reservoir properties", in: Distributed Parameter Systems: Identification, Estimation and Control (ed. W. H. Ray amd D. G. Lainiotis) 
(Marcel Dekker, New York, 1978).

[18] W. H. Yu, "A Rapid convergent iteration method for identifying distributed parameters in partial differential equations", Preprints 1988 'IFAC Symp. Identification and System Parameter Estimation (Beijing, 1988).

[19] W. H. Yu and J. H. Seinfeld, "Identification of distributed paremeter systems by regularization with differential operator", J. Math. Anal. Appl. 132 (1988) 365-398.

[20] W. H. Yu and J. H. Seinfeld, "Identification of distributed parameter systems with pointwise constrains on the parameters", J. Math. Anal. Appl. 136 (1988) 497-520.

[21] W. H. Yu, "Solving inverse problems for hyperbolic equations via the regularization method", J. Computational Mathematics 11 (1993) 142-153.

[22] Jianping Zhu and Yung Ming Chen, "Multilevel grid method for history matching multidimensional multi-phase reservoir models", Applied Numer. Math. 10 (1992) 159-174. 